\title{
Lipidic phase membrane protein serial femtosecond crystallography
}

Linda C. Johansson ${ }^{1}$, David Arnlund ${ }^{1}$, Thomas A. White ${ }^{2}$, Gergely Katona ${ }^{1}$, Daniel P. DePonte $^{2}$, Uwe Weierstall ${ }^{3}$, R. Bruce Doak ${ }^{3}$, Robert L. Shoeman ${ }^{4}$, Lukas Lomb ${ }^{4}$, Erik Malmerberg $^{1}$, Jan Davidsson ${ }^{5}$, Karol Nass ${ }^{6}$, Mengning Liang ${ }^{2}$, Jakob Andreasson ${ }^{7}$, Andrew Aquila $^{2}$, Saša Bajt ${ }^{2}$, Miriam Barthelmess ${ }^{8}$, Anton Barty ${ }^{2}$, Michael J. Bogan ${ }^{9}$, Christoph Bostedt $^{10}$, John D. Bozek ${ }^{10}$, Carl Caleman ${ }^{2}$, Ryan Coffee $^{10}$, Nicola Coppola $^{2,18}$, Tomas Ekeberg $^{7}$, Sascha W. Epp ${ }^{11,12}$, Benjamin Erk $^{11,12}$, Holger Fleckenstein ${ }^{2}$, Lutz Foucar ${ }^{4,11}$, Heinz Graafsma $^{8}$, Lars Gumprecht ${ }^{2}$, Janos Hajdu ${ }^{7}$, Christina Y. Hampton ${ }^{9}$, Robert Hartmann ${ }^{13}$, Andreas Hartmann ${ }^{13}$, Günter Hauser ${ }^{14}$, Helmut Hirsemann ${ }^{8}$, Peter Holl ${ }^{13}$, James M. Holton ${ }^{15}$, Mark S. Hunter ${ }^{16}$, Stephan Kassemeyer ${ }^{4}$, Nils Kimmel ${ }^{14}$, Richard A. Kirian ${ }^{3}$, Filipe R.N.C. Maia $^{15}$, Stefano Marchesini ${ }^{15}$, Andrew V. Martin ${ }^{2}$, Christian Reich ${ }^{13}$, Daniel Rolles ${ }^{4,11}$, Benedikt Rudek $^{11,12}$, Artem Rudenko ${ }^{11,12}$, Ilme Schlichting ${ }^{4,11}$, Joachim Schulz ${ }^{2}$, M. Marvin Seibert $^{7,19}$, Raymond Sierra ${ }^{9}$, Heike Soltau ${ }^{13}$, Dimitri Starodub ${ }^{9}$, Francesco Stellato ${ }^{2}$, Stephan Stern $^{2}$, Lothar Strüder ${ }^{11,14}$, Nicusor Timneanu ${ }^{7}$, Joachim Ullrich ${ }^{11,12}$, Weixiao Y. Wahlgren ${ }^{1}$, Xiaoyu Wang ${ }^{3}$, Georg Weidenspointner ${ }^{14,17}$, Cornelia Wunderer ${ }^{8}$, Petra Fromme $^{16}$, Henry N. Chapman $^{2,6}$, John C. H. Spence ${ }^{3}$, Richard Neutze ${ }^{1}$. 
1. Department of Chemistry and Molecular Biology, University of Gothenburg,

Gothenburg, Sweden.

2. Center for Free-Electron Laser Science, Deutsches Elektronen-Synchrotron, Hamburg, Germany.

3. Department of Physics, Arizona State University, Tempe, AZ, USA.

4. Max-Planck-Institut für Medizinische Forschung, Heidelberg, Germany

5. Department of Photochemistry and Molecular Science, Uppsala University, Uppsala, Sweden.

6. University of Hamburg, Hamburg, Germany.

7. Laboratory of Molecular Biophysics, Department of Cell and Molecular Biology, Uppsala University, Uppsala, Sweden.

8. Photon Science, Deutsches Elektronen-Synchrotron, Hamburg, Germany.

9. PULSE Institute, SLAC National Accelerator Laboratory, Menlo Park, CA, USA.

10. Linac Coherent Light Source, SLAC National Accelerator Laboratory, Menlo Park, CA, USA.

11. Max Planck Advanced Study Group, Center for Free Electron Laser Science, Hamburg, Germany.

12. Max-Planck-Institut für Kernphysik, Heidelberg, Germany.

13. PNSensor GmbH, München, Germany.

14. Max-Planck-Institut Halbleiterlabor, München, Germany.

15. Advanced Light Source, Lawrence Berkeley National Laboratory, Berkeley, CA, USA.

16. Department of Chemistry and Biochemistry, Arizona State University, Tempe, AZ, USA.

17. Max-Planck-Institut für Extraterrestrische Physik, Garching, Germany.

18. Present address: European XFEL GmbH, Hamburg, Germany. 
19. Present address: Linac Coherent Light Source, SLAC National Accelerator Laboratory,

Menlo Park, CA, USA.

Corresponding author: richard.neutze@chem.gu.se 
X-ray free electron laser (X-FEL) based serial femtosecond crystallography is an emerging method with potential to rapidly advance the challenging field of membrane protein structural biology. Here we adapted the lipidic sponge phase crystallization of membrane proteins to be compatible with serial femtosecond crystallography. We recorded interpretable diffraction data from micrometer-sized lipidic sponge phase crystals of the Blastochloris viridis photosynthetic reaction center delivered into an $\mathrm{X}$ FEL beam using micro-jet technology.

Membrane proteins remain a major challenge within structural biology. They contain both hydrophobic and hydrophilic surfaces, are typically scarce and highly flexible, and frequently become unstable when removed from their natural membrane environment. These characteristics create substantial obstacles for growing large well ordered crystals suitable for conventional crystallography ${ }^{1}$. Thus the recent demonstration of serial femtosecond crystallography ${ }^{2}$ holds great promise for the structural analysis of membrane proteins since it makes it possible to recover high resolution X-ray diffraction data from thousands of submicron to micron sized crystals.

Serial femtosecond crystallography was first demonstrated ${ }^{2}$ using sub-micron crystals of cyanobacterial photosystem I (PSI) delivered to the X-ray beam using a thin liquid jet ${ }^{3}$. As a large membrane protein complex, PSI is exceptional in that it crystallizes overnight at low ionic strength ${ }^{4}\left(8 \mathrm{mM} \mathrm{MgSO}_{4}\right)$. More representative membrane protein crystallization conditions involve higher concentrations of salt (typically 50 to $300 \mathrm{mM}$ ) and PEG (typically 10 to $35 \%$ ) which pose challenges when using micro-jet injection due to higher viscosity and the risk that salt crystals or aggregates may clog the micro jet nozzle. 
Lipidic cubic phase (LCP) crystallization of membrane proteins ${ }^{5-7}$ is a generic crystallization method developed to mimic the natural lipidic bilayer of membrane proteins and thereby enhance their stability during crystallization. In the original formulation of LCP crystallization $^{5}$, solubilized membrane proteins were first mixed with the lipid monoolein in the ratio 60:40 to form the semi-solid LCP, to which crystallization agents were then added. LCP crystallization initially led to rapid advances with the structural biology of archaeal rhodopsins and more recently concerning the structural biology of G-protein coupled receptors (Supplementary Table 1).

Because of its semi-solid nature, the LCP does not readily form a micron sized jet required to deliver microcrystals in serial femtosecond crystallography ${ }^{2}$. On the other hand, the closely related lipidic sponge phase (LSP) presents a liquid lipid environment that can be adapted to this new approach to structural biology. As with LCP, the LSP is made by mixing monoolein and water, but with the addition of a third agent such as jeffamine or PEG that swells the cubic phase into a liquid phase $\mathrm{e}^{7,8}$. LSP crystallization was originally developed from the observation that the LCP crystallization of the Rhodobacter sphaeroides reaction centre proceeded via a LSP ${ }^{8}$. Four independent LSP membrane protein structures have been reported to date (Supplementary Table 1), it appears likely that crystals used for determining the structures of several G-protein coupled receptors and one bacterial oxidase grew via a cubicto-sponge-phase transition (Supplementary Note), and a LSP crystallization screen gave leads from eight of eleven membrane proteins tested ${ }^{9}$. Thus LCP and LSP crystallization are closely related, generic approaches to membrane protein structural biology. In this work we adapted the LSP crystallization to yield showers of membrane protein microcrystals that we injected across an X-ray free electron beam using micro-jet technology ${ }^{3}$. From the diffraction 
data we recovered a new crystal form of the Blastochloris viridis photosynthetic reaction centre $\left(\mathrm{RC}_{v i r}\right)$ solved using serial femtosecond crystallography.

We set up batch LSP crystallization experiments using $250 \mu 1$ aliquots in septum-sealed glass vials (Fig. 1a). These crystallization conditions we adapted from earlier hanging-drop conditions ${ }^{10}$ with minor modifications (online Methods) after initially screening for crystal showers using smaller batch crystallization setups. Since the sponge phase is less dense than water, it spontaneously separates ${ }^{8}$, with the LSP floating on the top (Fig. 1a, colored brown). It is within this phase that membrane protein microcrystals grew. We used optical microscopy to identify crystallization conditions under which showers of crystals grew over a period of two weeks (Fig. 1b) and cross-polarization confirmed their ordered nature through birefringence (Supplementary Fig. 1).

These crystallization setups we shipped at room temperature to the Linac Coherent Light Source (LCLS) at SLAC National Laboratory, USA. We collected the colored upper phase (Fig. 1a) immediately prior to injection into the X-FEL beam and large crystals, which could potentially block the micro-jet, we removed by passing the LSP through a $10 \mu \mathrm{m}$ cutoff metal filter (online Methods). Suspensions of filtered crystals we injected as a rapidly flowing liquid micro-jet ${ }^{3}$ (Supplementary Fig. 2) across a focused X-FEL beam ${ }^{11}$. The sponge phase (upper layer, Fig. 1a) readily flowed at a rate similar to that of water $(10 \mu \mathrm{l} / \mathrm{min})$ and produced a stable jet approximately $4 \mu \mathrm{m}$ in diameter (Fig. 2a). Since the diameter of this jet was of the same order of magnitude as the $\mathrm{RC}_{v i r}$ microcrystals, the LSP provided a lowbackground environment well suited for the delivery of microcrystals for serial femtosecond crystallography. 
We collected diffraction data at the Atomic Molecular and Optical (AMO) beamline ${ }^{12}$ of the LCLS on the CAMP p-n junction charge-coupled devices ${ }^{13}$ (pnCCDs) with two vertically offset panels that we read out at $60 \mathrm{~Hz}$, which was the repetition rate of the FEL X-ray pulses. Each X-FEL pulse was nominally 70 fs in duration and contained up to $10^{13} 2 \mathrm{keV}$ X-rays $(\lambda$ $=6.17 \AA$ ) focused into a $10 \mu \mathrm{m}^{2}$ spot. The maximum resolution accessible with this energy and detector geometry was $7.4 \AA$ and reaction center microcrystals repeatedly diffracted to this resolution limit (Fig. 2b). Of the 365,035 recorded frames (approximately 100 minutes of data collection), we observed 1542 diffraction patterns of which 265 we processed in the space group $\mathrm{P} 2{ }_{1} 2_{1} 2_{1}$ (Fig. 2c) with unit cell axes $\mathrm{a}=57.6 \AA, \mathrm{b}=84.6 \AA, \mathrm{c}=375.8 \AA$ and $\alpha=$ $\beta=\gamma=90^{\circ}$ (Supplementary Fig. 3), representing a new crystal form of $\mathrm{RC}_{v i r}$.

We processed this diffraction data using Monte Carlo methods ${ }^{14,15}$ since each diffraction pattern recorded only partial reflections from randomly oriented microcrystals. Supplementary Table 2 summarizes the crystallographic data recovered from this analysis, with Supplementary Table 3 providing a breakdown of crystallographic statistics for each resolution shell. The best molecular replacement solution (online Methods) showed the crystals to pack as stacked layers of 2D crystals typically found in lipidic phase crystallization $^{7}$ (Supplementary Fig. 4). During structural refinement we cut the diffraction data to $8.2 \AA$ resolution, for which the multiplicity was greater than 4 and completeness above $95 \%$ (Supplementary Table 3). Structural refinement yielded $R_{\text {factor }}$ and $\mathrm{R}_{\text {free }}$ values of $35 \%$ and $38 \%$ respectively (Supplementary Table 2; Supplementary Table 4 provides a breakdown by resolution shell) and both the $2 \mathrm{~F}_{\text {obs }}-\mathrm{F}_{\text {calc }}$ electron density map (Fig. 3a) and composite omit map (Supplementary Fig. 5) clearly indicated transmembrane $\alpha$-helices. Moreover, when all four heme groups of the $\mathrm{RC}_{v i r}$ cytochrome subunit were removed from 
the structural model we recovered positive $\mathrm{F}_{\mathrm{obs}}-\mathrm{F}_{\text {calc }}$ electron density associated with each of these co-factors in the resulting omit map (Fig. 3b, Supplementary Fig. 6).

In this work we grew $\mathrm{RC}_{v i r}$ microcrystals in a LSP derived from monoolein, water and jeffamine. We also recovered well performing microjets using LSPs derived from monoolein, water and PEG 400, PEG 1500 or PEG 4000 (Supplementary Fig. 7). Since jeffamine and PEG conditions form the basis of a validated LSP crystallization screen ${ }^{9}$ and PEG 400 has been a crystallization agent in all recent LCP crystal structures of G-protein coupled receptors (Supplementary Table 1), the adaption of LSP crystallization to serial femtosecond crystallography appears promising for solving membrane protein targets of unknown structure.

We indexed only 265 diffraction patterns from LSP microcrystals of $\mathrm{RC}_{v i r}$, yielding a hit rate below $0.1 \%$ of the recorded images and approximately an order of magnitude lower than that obtained for $\mathrm{PSI}^{2}$. A probable explanation for this difference is that the $\mathrm{RC}_{v i r}$ microcrystals tended to have a high mosaic spread (Supplementary Fig. 8) which, in combination with one cell axis being longer than $300 \AA$, made data-processing challenging. Since PSI microcrystals were grown overnight on-site at the $\mathrm{LCLS}^{2}$ it is reasonable to expect that the quality of $\mathrm{RC}_{v i r}$ microcrystals would improve if long-distance shipping at room-temperature could be avoided. Other areas for improvement include more robust diffraction spot finding and indexing routines and slight changes in crystallization conditions so as to maximize the number of diffracting microcrystals.

Shorter X-ray wavelength beamlines $(\lambda \sim 1.5 \AA)$ and higher repetition rates $(120 \mathrm{~Hz})$ have recently become available at the LCLS. As such, high resolution membrane protein crystal 
structures should soon be achievable using X-FEL radiation. We estimate that the order of 10000 processed diffraction images will be needed for high-resolution electron density maps to be recovered using serial femtosecond crystallography, which would require three 12 hour shifts at the hit-rate reported here. Nevertheless, since it was possible to solve the structure of $\mathrm{RC}_{v i r}$ to $8.2 \AA$ resolution by molecular replacement with only 265 processed images, this bodes well for future applications of lipidic phase serial femtosecond crystallography to membrane protein structural biology. 


\section{METHODS}

Methods and any associated references are available in the online version of the paper at http://www.nature.com/naturemethods/.

\section{Accession codes.}

The crystal structure and diffraction data are available within the Protein Data Bank with accession code $4 \mathrm{ac} 5$.

Note: Supplementary information is available on the Nature Methods website.

\section{ACKNOWLEDGEMENTS}

Experiments were carried out at the Linac Coherent Light Source (LCLS), a national user facility operated by Stanford University on behalf of the U.S. Department of Energy (DOE), Office of Basic Energy Sciences. We gratefully acknowledge financial support from the Swedish science research council (VR), the Swedish Foundation for International Cooperation in Research and Higher Education (STINT), Stiftelsen Olle Engkvist Byggmästare, the Max Planck Society for funding the development and operation of the Center for Free-Electron Laser Science (CFEL) Advanced Study Group (ASG) Multi-Purpose Chamber (CAMP) instrument, the US National Science Foundation (NSF) grant MCB 0919195, the DOE Office of Basic Energy Sciences through the Photon Ultrafast Laser Science and Engineering Center (PULSE) Institute at the Stanford Linear Accelerator Center (SLAC) National Accelerator Laboratory and the Energy Frontier Research Center for Bio- Inspired Solar Fuel Production (award DE-SC0001016), the Hamburg Ministry of Science and Research and Joachim Herz Stiftung as part of the Hamburg Initiative for Excellence in Research (LEXI) and the Hamburg School for Structure and Dynamics in Infection, National Science Foundation 
(awards 0417142 and MCB-1021557), National Institute of Health (awards 1R01GM095583-

01 and 1U54GM094625-01), the Deutsche Forschungsgemeinschaft (DFG) Cluster of Excellence at the Munich Center for Advanced Photonics, Center for Biophotonics Science and Technology (CBST) at the University of California (UC) under Cooperative Agreement No. PHY 0120999.

\section{AUTHOR CONTRIBUTIONS}

L.C.J., R.N., H.N.C., J.C.H.S., P.F conceived the experiment which was designed with A.B., R.A.K., J.C.H.S., D.P.D., U.W., R.B.D., M.J.B., D.S., I.S., S.M. and J.H. The CAMP instrument was the responsibility of S.W.E., R.H., D.R., A.R., C.Sch, L.F., N.K., P.H., B.R., B.E., A.H., Ch.R., D.P., G.W., L.S., G.H., H.G., J.U., I.S., S.H., G.Sch., F.Sch., H.S., K.U.K., R.A., K.-D.S., F.K., Ma.B., S.Sch., D.Ru., M.A., T.G., H.H., L.G., G.P., H.G., and B.N. who designed and set up the instrument and/or developed and operated the pnCCD detectors. C.B., and J.B. set up and aligned the beamline.; L.C.J, D.A. grew sponge phase crystals; U.W, R.B.D., J.C.H.S. , D.P.D., R.L.S., L.L designed and operated the injector, H.N.C., A.B., A.A., J.S., D.P.D., U.W., R.B.D., S.Ba., M.J.B., L.G., J.H., M.M.S., N.T., J.A., S.St., and J.C.H.S. developed diffraction instrumentation, L.C.J., D.A., T.A.W., D.P.D., U.W., R.B.D., R.L.S., L.L., E.M., J.D., K.N., M.L., A.A., M.B., A.B., M.J.B., C.B., J.B., C.C., R.C., N.C., T.E., H.F, P.F., C.Y.H., J.M.H., M.S.H., S.K., R.A.K., F.M., A.V.M., I.S., M.M.S., R.S., F.S., N.T., X.W., C.W., H.N.C., J.C.H.S., and R.N. collected data. L.C.J., D.A., T.A.W., G.K., W.Y.W., and K.N. analyzed the diffraction data using software developed by J.C.H.S, T.A.W., R.K., A.B., and H.N.C. L.C.J. and R.N wrote the paper with discussion and improvements from all authors.

\section{COMPETING FINANCIAL INTERESTS}


The authors declare no competing financial interests. 


\section{References}

1 Bill, R. M. et al. Nat. Biotechnol. 29, 335-340 (2011).

2 Chapman, H. N. et al. Nature 470, 73-77 (2011).

3 DePonte, D. P. et al. J. Phys. D: Appl. Phys. 41, 195505 (2008).

$4 \quad$ Jordan, P. et al. Nature 411, 909-917 (2001).

5 Landau, E. M. \& Rosenbusch, J. P. Proc. Natl. Acad. Sci. USA 93, 14532-14535 (1996).

6 Caffrey, M. Annu. Rev. Biophys. 38, 29-51 (2009).

7 Johansson, L. C., Wohri, A. B., Katona, G., Engstrom, S. \& Neutze, R. Curr. Opin. Struct. Biol. 19, 372-378 (2009).

8 Wadsten, P. et al. J. Mol. Biol. 364, 44-53 (2006).

9 Wöhri, A. B. et al. Structure 16, 1003-1009 (2008).

10 Wohri, A. B. et al. Biochemistry 48, 9831-9838 (2009).

11 Emma, P. et al. Nat. Photon. 4, 641-647 (2010).

12 Bozek, J. D. Eur. Phys. J.Special Topics 169, 129-132 (2009).

13 Strüder, L. et al. Nuc. Inst. Meth. Phys. Res. A. 614, 483-496 (2010).

14 Kirian, R. A. et al. Opt. Express. 18, 5713-5723 (2010).

15 Kirian, R. A. et al. Acta Crystallogr. A 67, 131-140 (2011). 


\section{Captions to Figures:}

Figure 1: Lipidic sponge phase batch crystallization of $\mathrm{RC}_{v i r}$. (a) $250 \mu 1$ batch crystallization setup in a glass vial. The sponge phase containing $\mathrm{RC}_{\text {vir }}$ (brown) floats to the top. (b) Optical microscopy image of the sponge phase setups indicating showers of crystals. Larger crystals are approximately $20 \mu \mathrm{m}$ long.

Figure 2: Serial femtosecond crystallography of $\mathrm{RC}_{v i r}$ crystals grown in a LSP. (a) Liquid jet formed by the sponge phase containing $\mathrm{RC}_{v i r}$ crystals. The $\mathrm{X}-\mathrm{FEL}$ beam interacting with the liquid jet is visible as a fluorescent spot. (b) Bragg diffraction spots (dark spots) recorded from a single $\mathrm{RC}_{v i r}$ crystal using a single $\mathrm{X}$-FEL pulse of $70 \mathrm{fs}$. (c) An identical diffraction image as shown in $\mathbf{b}$, but with the predicted spot positions after data indexing shown as circles. The resolution was limited to $7.4 \AA$ in the corners of the lower pnCCD detector panel.

Figure 3: Electron density for the LSP serial femtosecond crystallography $\mathrm{RC}_{v i r}$ structure at $8.2 \AA$ resolution. (a) Stereo view of the $2 \mathrm{mF}_{\mathrm{obs}}-\mathrm{DF}_{\text {calc }}$ electron density map (contoured at 1.0 $\sigma)$ recovered from 265 processed $\mathrm{RC}_{\text {vir }}$ diffraction images. (b) Stereo view of the $\mathrm{mF}_{\mathrm{obs}}-\mathrm{DF}_{\text {calc }}$ omit electron density map (contoured at $2.0 \mathrm{\sigma}$ ), calculated with the four heme groups of the cytochrome subunit removed from the structural model. This figure was generated with PYMOL (DeLano Scientific LLC). 


\section{ONLINE METHODS}

Growth and purification of reaction center from $B$ l. viridis. We cultivated the photosynthetic reaction center from $B l$. viridis as described ${ }^{10}$. We modified the purification by using $250 \mathrm{~mL}$ POROS 50 micron HQ media (Applied Biosystems Europe BV) packed in an XK 50/20 column (GE Healthcare) and a HiPrep 26/60 Sephacryl S-300 column (GE Healthcare). This protocol yielded approximately $3 \mathrm{mg}$ of pure $\mathrm{RC}_{v i r}$ per $\mathrm{L}$ cell culture.

Lipidic sponge phase batch crystallization. We prepared lipidic sponge phases as previously described ${ }^{10}$. We set up batch crystallizations in septum-sealed glass vials (SigmaAldrich) containing $100 \mu \mathrm{l}$ protein $(20-30 \mathrm{mg} / \mathrm{ml}), 100 \mu \mathrm{l}$ lipidic sponge phase $(12 \%$ Monoolein, 17.5 \% Jeffamine M-600, 1.0 M Hepes pH 8.0, $0.7 \mathrm{M}\left(\mathrm{NH}_{4}\right)_{2} \mathrm{SO}_{4}, 2.5 \%$ 1,2,3heptanetriol) and $50 \mu 1$ 1.0-1.2 M Tri-sodium citrate. These setups were left to equilibrate for two to four weeks at $20^{\circ} \mathrm{C}$. We diluted crystals $4: 1$ in a solution containing $0.1 \mathrm{M}$ Hepes $\mathrm{pH}$ 8.0, $0.1 \%$ LDAO prior to filtering in a $10 \mu \mathrm{m}$ cutoff titanium filter (VICI AG International). We developed the conditions for large-batch crystallization setups by initially screening for batch crystallization conditions using smaller batch crystallization volumes (60 $\mu 1$ to $100 \mu 1$ setups).

Liquid microjet. We delivered samples to the injector nozzle via a sample loop and we injected these into the X-FEL beam at a flow rate of $10 \mu 1 / \mathrm{min}$. The liquid capillary of the nozzle had an inner diameter of $50 \mu \mathrm{m}$ and the liquid was focused by coaxially flowing helium gas to a continuous jet-stream of about $4 \mu \mathrm{m}$ diameter $^{3}$. We aligned the X-ray beam to hit the liquid in the continuous jet region before the breakup into droplets occurred (Fig. 2a). For PEG based LSPs, we required an inner diameter of $100 \mu \mathrm{m}$ for the jet to flow (Supplementary Fig. 7). These LSPs consisted of $40 \%$ PEG400, PEG 1500 or PEG4000 
mixed with $30 \%$ Monoolein and $30 \%$ buffer $(0.1 \mathrm{M}$ Hepes, $\mathrm{pH} 8.0,0.1 \mathrm{M} \mathrm{NaCl}$ and $0.1 \mathrm{M}$ $\left.\mathrm{MgCl}_{2}\right)$

Data collection. We collected diffraction data at the Atomic Molecular and Optical (AMO) beamline at the Linac Coherent Light Source ${ }^{12}$ using the CFEL-ASG Multi-Purpose (CAMP) instrument ${ }^{13}$. We recorded diffraction data on two $76.8 \mathrm{~mm}$ by $38.4 \mathrm{~mm}$ pnCCDs located 64.7 $\mathrm{mm}$ and $67.7 \mathrm{~mm}$ from the sample position. The pnCCDs we offset asymmetrically such that X-ray diffraction was sampled from $3.5^{\circ}$ to $49.0^{\circ}$, which maximized the sampled resolution (7.4 $\AA$ at the outer corners of the lower panel, Fig. 2b). The X-ray wavelength at AMO was $6.17 \AA$ and we focused the beam to a $10 \mu \mathrm{m}^{2}$ spot.

Data processing. We processed diffraction data using in-house code that called DirAx ${ }^{16}$ and $\operatorname{MOSFLM}^{17}$ for automated indexing ${ }^{2}$. We indexed data from 265 diffraction images in $\mathrm{P} 2{ }_{1} 2{ }_{1} 2_{1}$ with unit cell axes $\mathrm{a}=57.6 \AA, \mathrm{b}=84.6 \AA, \mathrm{c}=375.8 \AA$ and $\alpha=\beta=\gamma=90^{\circ}$. We did not observe sub-populations of other crystal forms (Supplementary Fig. 3). Small variations in the length of the c-axis, however, arose perhaps due to pulse-to-pulse fluctuations in the XFEL wavelength. Since every observation is a partial reflection, we scaled and merged this integrated data using Monte Carlo methods ${ }^{14,15}$. We estimated $I / \sigma$ values for each resolution bin (Supplementary Table 3) from reflections with both positive intensity and a multiplicity higher than 1. NZ-test and L-test plots showed the expected distributions (Supplementary Fig. 9).

Molecular replacement and refinement. We obtained phases by molecular replacement using Phaser ${ }^{18}$ 2.3.0 with PDB entry $2 \mathrm{WJN}^{10}$ as the search model. The best solution had a translation function Z-score (TFZ) of 8.5, the rotation function Z-score (RFZ) of 5.8, and a 
log likelihood gain (LLG) of 81. This was well discriminated from second best solution with scores of TFZ: 4.6, RFZ: 5.8, and LLG: 39 . We evaluated the crystal packing and confirmed that only the best molecular replacement solution was physically meaningful. We performed twenty cycles of rigid body and restrained refinement using REFMAC ${ }^{19}$, converging to $\mathrm{R}_{\text {factor }}$ $=35 \%$ and $\mathrm{R}_{\text {free }}=38 \%$, and an overall figure of merit of 0.62 . During refinement we used simple Wilson scaling and a constant density (using default values) we assigned to the region of the unit cell not occupied by protein atoms. We calculated the solvent mask using default parameters: increase VDW radius of non-ion atoms by $1.2 \AA$, increase ionic radius of potential ions by $0.8 \AA$, shrink the area of the mask by $0.8 \AA$ after calculation. Crystallographic data statistics are summarized in Supplementary Table 2, and resolution shell breakdowns of the crystallographic data and refinement statistics are given in Supplementary Tables 3 and 4 respectively.

Control map calculations. We also performed test calculations using two control datasets: one with the serial femtosecond crystallography data randomly shuffled and another where all observations were set equal. Molecular replacement failed with both control data-sets. When we combined the phases generated using the molecular replacement solution recovered against the experimental data with these control data sets, the resulting electron density maps did not show $\alpha$-helical structure (Supplementary Fig. 10).

Omit map calculations. We calculated $\mathrm{F}^{\text {obs }}-\mathrm{F}^{\text {calc }}$ omit electron density maps using REFMAC $^{19}$ with all four hemes of the cytochrome subunit removed from the structural model (Fig. 3b; Supplementary Fig. 6). 
Composite omit map calculations. We calculated composite omit electron density maps (Supplementary Fig. 5) using $\mathrm{CNS}^{20,21}$ version 1.3 with approximately $5 \%$ of the structure excluded, no simulated annealing, no minimization and no bulk solvent correction.

16 Duisenberg, A. J. M. J. Appl. Cryst. 25, 92-96 (1992).

17 Leslie, A. G. W. Joint CCP4 + ESF-EAMCB Newsletter on Protein Crystallography, No. 26. (1992).

18 McCoy, A. J. et al. J. Appl. Cryst. 40, 658-674 (2007).

19 Murshudov, G. N., Vagin, A. A. \& Dodson, E. J. Acta. Crystallogr. D53, 240-255 (1997)

20 Brunger, A. T. et al. Acta Crystallogr. D Biol. Crystallogr. 54, 905-921 (1998).

21 Brunger, A. T. Nat. Protoc. 2, 2728-2733 (2007). 


\begin{tabular}{|c|c|}
\hline Supplementary File & Title \\
\hline Supplementary Figure 1 & Cross-polarization microscopy. \\
\hline Supplementary Figure 2 & Schematic of experimental protocol. \\
\hline Supplementary Figure 3 & Cell axes histograms. \\
\hline Supplementary Figure 4 & Crystal packing. \\
\hline Supplementary Figure 5 & Composite omit electron density maps. \\
\hline Supplementary Figure 6 & Electron density maps with hemes omitted. \\
\hline Supplementary Figure 7 & PEG-based LSP microjet images. \\
\hline Supplementary Figure 8 & Diffraction image from a mosaic crystal. \\
\hline Supplementary Figure 9 & NZ-test and L-test plots. \\
\hline Supplementary Figure 10 & Electron density maps against control data. \\
\hline Supplementary Table 1 & LCP and LSP crystal structures. \\
\hline Supplementary Table 2 & X-ray data and refinement statistics. \\
\hline Supplementary Table 3 & X-ray data statistics by resolution shell. \\
\hline Supplementary Table 4 & Refinement statistics by resolution shell. \\
\hline \multicolumn{2}{|l|}{ Supplementary Note } \\
\hline Supplementary References & \\
\hline
\end{tabular}


a

$200 \mu \mathrm{m}$

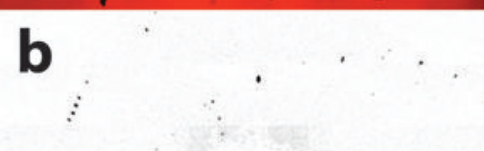

1

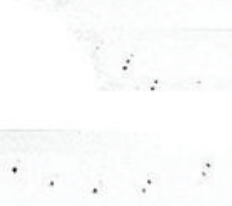

c

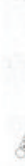

8
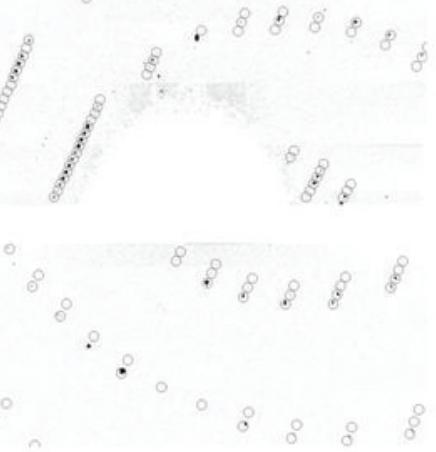
